\title{
Optical Luminosity Function of Quasi Stellar Objects
}

\author{
Salam Ajitkumar Singh ${ }^{1,}$, Irom Ablu Meitei ${ }^{2}$, Kangujam Yugindro Singh ${ }^{1}$ \\ ${ }^{1}$ Department of Physics, Manipur University, Imphal, India \\ ${ }^{2}$ Department of Physics, Modern College, Imphal, India
}

Email address:

ajitkumarsalam@gmail.com (S. A. Singh)

${ }^{*}$ Corresponding author

\section{To cite this article:}

Salam Ajitkumar Singh, Irom Ablu Meitei, Kangujam Yugindro Singh. Optical Luminosity Function of Quasi Stellar Objects. American Journal of Astronomy and Astrophysics. Vol. 4, No. 6, 2016, pp. 78-82. doi: 10.11648/j.ajaa.20160406.12

Received: October 29, 2016; Accepted: November 11, 2016; Published: December 8, 2016

\begin{abstract}
We study the shape of the optical luminosity function of Quasi Stellar Objects (QSOs) from the Sloan Digital Sky Survey Data Release Seven (SDSS DR7) over the redshift range $0.3 \leq z \leq 2.4$. By using the Levenberg-Marquardt method of nonlinear least square fit, the observed QSO luminosity function is fitted by a double power-law model with luminosity evolution characterized by a second order polynomial in redshift. For a flat universe with $\Omega_{\mathrm{m}}=0.3$ and $\Omega_{\Lambda}=0.7$, we determine the best-fitting optical luminosity function model parameters.
\end{abstract}

Keywords: Quasars: General, Quasars: Supermassive Black Holes, Galaxies: Luminosity Function

\section{Introduction}

Quasi Stellar Objects (QSOs) or quasars are intrinsically luminous subclass of Active Galactic Nuclei (AGNs) and these objects represent a fascinating and unique population of objects at the intersection of cosmology and astrophysics [1]. Soon after the discovery of QSOs their population was observed to be evolving [2]. As a result these objects provide a unique tool in the study of galaxies and large-scale structure formation throughout the history of the universe [3]. The luminosity function of QSOs describes the distribution of these objects in space as a function of their luminosity. Such a function also depends on other properties e.g. environment, evolutionary stage of the universe etc. [4]. If one specifies the dependence of mass on luminosity one can use the luminosity function to determine the local mass density [5]. Due to strong evolution of QSOs with cosmic time the luminosity function of the QSOs must be of particular importance in the understanding of formation and evolution of the QSOs. The QSO luminosity function and its evolution with redshift are the most important tools to constrain the accretion history of supermassive black holes (SMBHs) [6] and provide important clues about the demographics of the AGN population and constraints on physical models and evolutionary theories of AGN [7-9]. The faint-end slope of the luminosity function is a measure of how much time QSOs spend at relatively low accretion rates. The bright-end slope, on the other hand, tells us about the intrinsic properties of the QSO population during the time when black holes were increasing in mass most rapidly [10]. The QSOs are also strong X-ray sources, thus the QSO luminosity function can provide important constraints on the contribution of QSOs to the X-ray and UV background radiation [11-14].

The QSO luminosity function provides an essential constraint on how the population characteristics have changed with time [11]. The differential quasar luminosity function is defined as the number of quasars per unit comoving volume, per unit luminosity as a function of luminosity and redshift $[15,16]$. The luminosity function of QSOs is usually calculated by the classical $1 / V_{\max }$ method (e.g. [17-20]). The most common analytical representation for the shape of QSO luminosity function in the literature is a double power-law model (e.g. [1,21-24]). The Schechter function model [25] can also be used to represent the shape of QSO luminosity function in the previous papers such as Goldschmidt \& Miller (1998) [26], Warren et al. (1994) [27], Singh \& Singh (2016) [28] and Singh et al. (2016) [29].

The evolution of the luminosity function can be generally modeled as pure luminosity evolution (PLE) or pure density evolution (PDE) [2]. Under the PLE scenario the number density of QSOs remains constant with redshift, but their luminosities change with time and under the PDE scenario the 
number density of QSOs changes but their luminosities remain constant. More complex models are also used to describe the evolution of luminosity function namely the Luminosity Dependent Density Evolution (LDDE) and the Luminosity Evolution and Density Evolution (LEDE). Ross et al. (2013) [1] and Croom et al. (2009) [10] presented the LEDE where the bright-end and faint-end slopes have fixed values and normalization and characteristic luminosity evolve independently. Croom et al. (2009) [10] and Bongiorno (2007) [30] used the LDDE to study the evolution of QSO luminosity function. In this paper, the luminosity evolution with redshift is described by PLE. Throughout this paper we use a flat universe with $\Omega_{\mathrm{m}}=0.3, \Omega_{\Lambda}=0.7$ and $H_{0}=70.0 \mathrm{~km} \mathrm{~s}^{-1} \mathrm{Mpc}^{-1}$.

In section 2, we give a brief description of the SDSS sample. The determination of the optical luminosity function of QSOs and its statistical analysis are described in section 3 and in section 4 we discuss the comparison between the SDSS QSO luminosity function and some other surveys of QSO luminosity function. Finally, in section 5 we give our conclusions.

\section{The SDSS Survey}

The Sloan Digital Sky Survey (SDSS) [31] is an optical survey that has mapped more than 10,000 square degrees of sky located in the northern galactic hemisphere and partially along the Celestial Equator. The SDSS uses a dedicated wide field 2.5 $\mathrm{m}$ altitude-azimuth telescope [32] located at Apache Point Observatory (APO) near the Sacramento peak in Southern New Mexico. The telescope has $3^{0}$ diameter field of view, and it was equipped with a large-format moisac of $302048 \times 2048$ Tektronix CCD cameras [33] that took the images in five photometric bands: $\mathrm{u}, \mathrm{g}, \mathrm{r}, \mathrm{i}$ and $\mathrm{z}[33,34]$. The survey dataprocessing software measures the properties of each detected object in the imaging data in all five photometric bands and determines and applies both astrometric and photometric calibrations [35-37]. Photometric calibration is provided by simultaneous observations with a 20 inch $(0.51 \mathrm{~m})$ telescope at the same site $[35,38]$. The photometric system is based on the
$\mathrm{AB}$ magnitude scale [39] and the photometric measurements are reported as asinh magnitudes [40].

The SDSS DR7 quasar catalog is described in [41]. It consists of 105,783 spectroscopically confirmed quasars that are brighter than $M_{i}=-22.0$, have at least one broad emission line (FWHM> $1000 \mathrm{~km} \mathrm{~s}^{-1}$ ), and have highly reliable redshifts [41]. The redshift distribution for SDSS DR7 quasars is also shown in Singh et al. (2014) [42]. About half of these objects were targeted by using the final quasar target selection algorithm described in [43], and form a homogeneous, statistical quasar sample. In this homogenous sample, quasars are flux-limited to $i=19.1$ for $z<2.9$ and to $i=20.2$ for $z>2.9$. The sky coverage of this uniform quasar sample is $6248 \mathrm{deg}^{2}$ [44].

\section{The QSO Optical Luminosity Function and Model Fitting}

The luminosity function of QSOs is determined by using the $1 / V_{\text {max }}$ method [2]. It is given by

$$
\Phi\left(M_{i}\right)=\frac{1}{\Delta M_{i}[z=2]} \sum_{j=1}^{N}\left(\frac{1}{V_{\max , j}}\right)
$$

and its Poisson statistical uncertainty is

$$
\sigma(\Phi)=\frac{1}{\Delta M_{i}[z=2]} \sqrt{\sum_{j=1}^{N}\left(\frac{1}{V_{\max , j}}\right)^{2}},
$$

where $V_{\max , j}$ is the comoving volume within which the $j^{\text {th }}$ object would be included in the sample. The summation is over all quasars within a redshift-magnitude bin.

The QSO luminosity function is calculated at absolute magnitude intervals of $0.3 \mathrm{mag}$ in nine separate redshift bins over the redshift range $0.3 \leq \mathrm{z} \leq 2.4$. The edges of the nine separate redshift bins are: $0.3,0.5,0.7,0.9,1.1,1.3,1.5,1.7$, 1.9 and 2.4. The resulting QSO luminosity function, calculated for a flat universe with $\Omega_{\mathrm{m}}=0.3, \Omega_{\Lambda}=0.7$ and $H_{0}=70.0 \mathrm{~km} \mathrm{~s}^{-1}$ $\mathrm{Mpc}^{-1}$, is shown in Figure 1 (filled circle points).

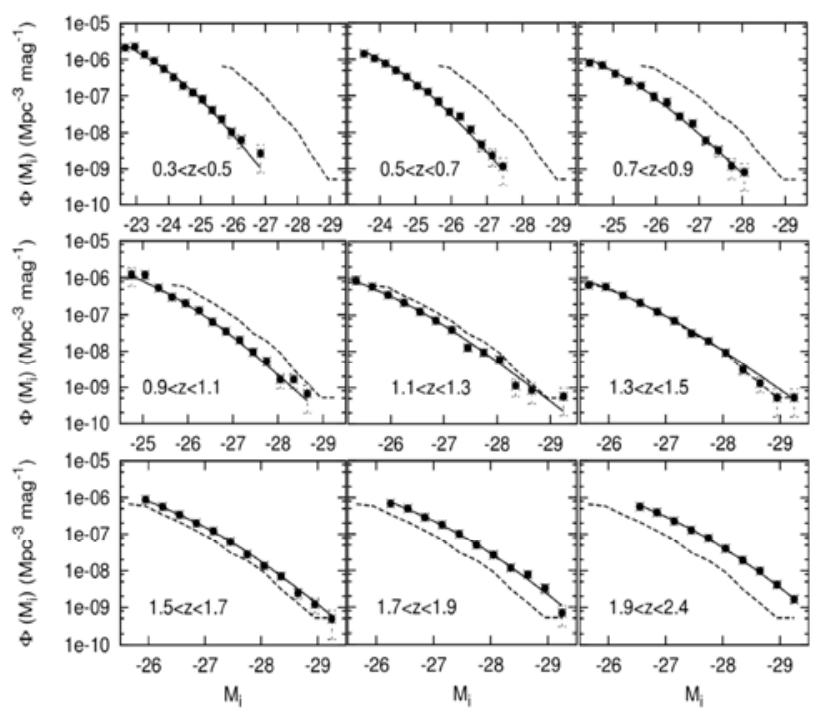

Figure 1. The optical luminosity functions of QSOs for the SDSS DR7 sample (filled circle points in each panel). The black solid lines represent the best fitting double power-law model with second order polynomial evolution. The dashed line in each panel shows the luminosity function at $1.3 \leq z \leq 1.5$ as a reference. 
The double power-law is used to fit the observed QSO luminosity function. The parametric form of the double powerlaw model, expressed in absolute magnitude, is given by

$$
\Phi\left(M_{i}, z\right)=\frac{\Phi^{*}}{\left[10^{0.4(\alpha+1)\left(M_{i}-M_{i}^{*}\right)}+10^{0.4(\beta+1)\left(M_{i}-M_{i}^{*}\right)}\right]},
$$

where $\alpha$ and $\beta$ are respectively the faint-end and bright-end slopes of the luminosity function. $M_{i}^{*}$ is the characteristic or break absolute magnitude. The evolution of the luminosity function is described by the redshift dependence of the characteristic magnitude $\left(M_{i}^{*}\right)$ which is chosen as a secondorder polynomial in redshift such that

$$
M_{i}^{*}(z)=M_{i}^{*}(0)-2.5\left(k_{1} z+k_{2} z^{2}\right)
$$

Using equations (3) and (4), we fit the PLE model to the observed luminosity function of QSOs in various redshift ranges. The best fit parameter values are estimated by using the Levenberg-Marquardt method of non linear least square fit [45]. The resulting best-fitting parameters in various redshift ranges are given in Table 1. The double power-law model with PLE fitted to the observed luminosity function of QSOs is shown in Figure 1 (denoted by solid lines). A $\chi^{2}$ comparison between the model luminosity function to the observed luminosity function gives $\chi^{2} / v=351.5 / 108$ for $0.3 \leq z \leq 2.4$. But, if we restrict the redshift range being fit, there is significant improvement to the fit with $\chi^{2} / v=78.9 / 40$ for the redshift range $1.3 \leq z \leq 2.4$ and $\chi^{2} / v=32.9 / 27$ for $1.5 \leq z \leq 2.4$.

Table 1. The best fitting parameter values for the double power-law with second order polynomial evolution in redshift.

\begin{tabular}{llllllll}
\hline $\begin{array}{l}\text { Redshift } \\
\text { Ranges }\end{array}$ & $\beta$ & $\alpha$ & $\boldsymbol{M}_{\boldsymbol{i}}^{*}$ & $\boldsymbol{k}_{1}$ & $\boldsymbol{k}_{2}$ & $\begin{array}{l}\Phi^{*} \times 10^{-07} \\
\boldsymbol{M p c}^{-3} \boldsymbol{m a g}^{-1}\end{array}$ & $\chi^{2} / \boldsymbol{v}$ \\
\hline $1.5-2.4$ & -4.19 & -2.44 & -22.58 & 1.91 & -0.44 & 1.31 & $32.9 / 27$ \\
$1.3-2.4$ & -3.91 & -2.11 & -22.28 & 1.83 & -0.42 & 3.51 & $78.9 / 40$ \\
$0.3-2.4$ & -3.89 & -2.18 & -23.44 & 1.36 & -0.29 & 2.93 & $351.5 / 108$ \\
\hline & & & \multicolumn{2}{c}{$M_{i}(z=2)=M_{b_{J}}-0.66 \pm 0.31[10,18]$. In Figure 2, the }
\end{tabular}

\section{Comparison to other Luminosity Functions}

In Figure 2, we compare the SDSS DR7 luminosity function to other luminosity functions calculated from Croom et al. 2004 (2QZ/6QZ) [22], Richards et al. 2005 (2SLAQ) [23] and Croom et al. 2009 (2SLAQ+SDSS) [10]. For comparision, we use the band transformations i.e. $M_{i}(z=$ 2) $=M_{g}(z=2)-0.255$

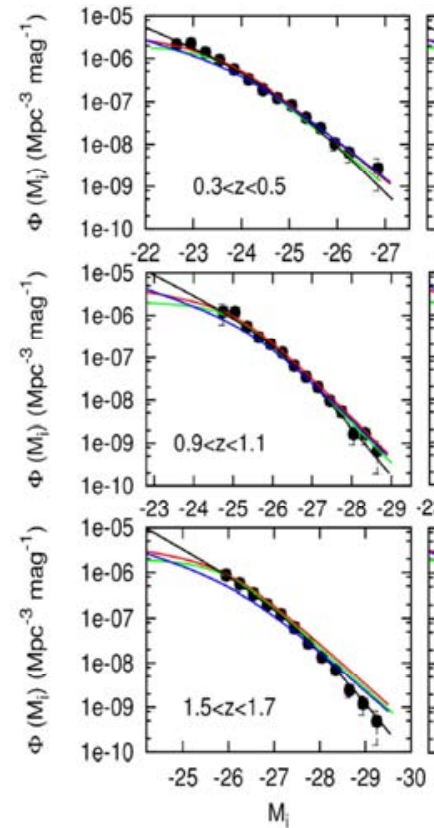

black lines in each panel are the best model fits to the SDSS DR7 QSO luminosity functions. The green, blue and red lines represent the best fitting double power-law model with a second order polynomial luminosity evolution from the 2QZ/6QZ, 2SLAQ and 2SLAQ+SDSS, respectively. In all redshift ranges, the SDSS DR7 predicts a higher density and steeper slope than the other surveys at the faint end of the luminosity function. At the bright end, the SDSS DR7 has slightly steeper slope of the luminosity function than the other surveys.

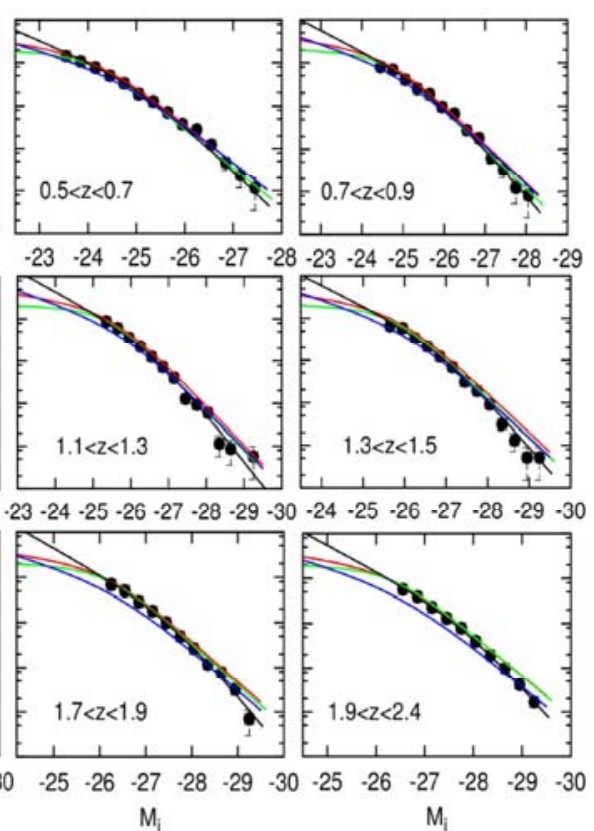

Figure 2. The double power-law model fit to the SDSS DR7 luminosity functions (black lines) compared to the best fit 2QZ/6QZ luminosity functions (green lines), 2SLAQ luminosity functions (blue lines) and $2 S L A Q+D R 3$ luminosity functions (red lines). 


\section{Conclusions}

The luminosity function of the QSOs in the SDSS DR7 and its evolution with redshift are studied by using the double power-law model with PLE. Croom et al. 2009 observed the double power-law with PLE to be relatively poor fit to the 2SLAQ luminosity function of QSOs over the redshift range $0.4 \leq z \leq 2.6$ with $\chi^{2} / v=256.6 / 81$. In our study, we obtain $\chi^{2} / v=351.5 / 108$ for the SDSS DR7 QSOs in the redshift range $0.3 \leq z \leq 2.4$. However, if we restrict the redshift range being fit, then we obtain significant improvement in the PLE model fit to the QSO luminosity functions with $\chi^{2} / v=32.9 / 27$ for the redshift range $1.5 \leq z \leq 2.4$. The six best fit parameters for the double power-law model with PLE are determined by using the Levenberg-Marquardt method of nonlinear least square fit. A Comparison of the $\chi^{2}$ values and the number of degrees of freedom shows that the double power-law model with polynomial evolution of the characteristic magnitude provides acceptable fit to the observed QSO luminosity function.

\section{Acknowledgments}

Funding for the SDSS and SDSS-II has been provided by the Alfred P. Sloan Foundation, the U.S. Department of Energy Office of Science, and the Participating Institutions. The SDSS Web site is http://www.sdss.org/.

The first author (Salam Ajitkumar Singh) is grateful to the Indian Space Research Organization, Department of Space, Government of India for providing JRF under a RESPOND Project (ISRO/RES/2/385/2013-14).

\section{References}

[1] N. P. Ross, et al., "The SDSS-III Baryon Oscillation Spectroscopic Survey: The quasar luminosity function from data release nine,” Astrophys. J., vol. 773, pp. 14, 2013.

[2] M. Schmidt, "Space distribution and luminosity functions of quasi-stellar radio sources," Astrophys. J., vol. 151, pp. 393, 1968.

[3] J. D. Kennefick, S. G. Djorgovski, R. R. De Carvalho, "The luminosity function of $z>4$ quasars from the second palomar sky survey,” Astron. J., vol. 110, pp. 2553, 1995.

[4] A. K. Kembhavi, J. V. Narlikar, "Quasars and Active Galactic Nuclei," Cambridge: Cambridge University Press, 1999.

[5] S. L. Shapiro, "The Density of Matter in the Form of Galaxies,” Astron. J. vol. 76. pp. 291, 1971.

[6] Q. Yu, S. Tremaine, "Observational constraints on growth of massive blackholes," Mon. Not. R. Astron. Soc., vol. 335, pp. 965-976, 2002.

[7] M. G. Haehnelt, M. J. Rees, "The formation of nuclei in newly formed galaxies and the evolution of the quasar population," Mon. Not. R. Astron. Soc., vol. 263, pp. 167-178, 1993.

[8] R. J. Terlevich, B. J. Boyle, "Young ellipticals at high redshift," Mon. Not. R. Astron. Soc., vol. 262, pp. 491-498, 1993.
[9] B. J. Boyle, et al., "The 2dF QSO Redshift Survey-I. The optical luminosity function of quasi-stellar objects," Mon. Not. R. Astron. Soc., vol. 317, pp. 1014-1022, 2000.

[10] S. M. Croom, et al., "The 2dF-SDSS LRG and QSO survey: the QSO luminosity function at $0.4<\mathrm{z}<2.6$," Mon. Not. R. Astron. Soc., vol. 399, pp. 1755-1772, 2009.

[11] D. C. Koo, and R. G. Kron, "Spectroscopic survey of QSOs to $\mathrm{B}=22.5$ : The luminosity function," Astrophys J., vol.325, pp. 92-102, 1988.

[12] B. J. Boyle, and R. J. Terlevich, "The cosmological evolution of the QSO luminosity density and of the star formation rate," Mon. Not. R. Astron. Soc., vol. 293, pp. L49-L51, 1998.

[13] R. F. Mushotzky, et al., "Resolving the extragalactic hard Xray background,"Nature, vol. 404, pp. 459, 2000.

[14] M. A. Worsley, et al., "The unresolved hard X-ray background: the missing source population implied by the Chandra and XMM-Newton deep fields," Mon. Not. R. Astron. Soc., vol. 357, pp. 1281-1287, 2005.

[15] L. Jiang, et al., "A spectroscopic survey of faint quasars in the SDSS DEEP STRIP. I. Preliminary results from the co-added catalog,” Astron. J., vol. 131, pp. 2788-2800, 2006.

[16] X. Fan, et al., "High-redshift quasars found in Sloan Digital Sky Survey commissioning data. IV. Luminosity function from the equatorial stripe sample," Astron. J.,vol. 121, pp. 5465, 2001.

[17] G. T. Richards, M. A. Strauss, X. Fan, et al. "The Sloan Digital Sky Survey Quasar Survey: Quasar luminosity function from data release 3," Astron. J., vol. 131, pp. 2766$2787,2006$.

[18] J. E. Greene, and L. C. Ho, "The Mass Function of Active Black Holes in Local Universe,” Astrophy. J., vol. 667, pp. 131-148, 2007.

[19] M. Vestergaard, and P. S. Osmer, "Mass functions of the active black holes in distant quasars from the Large Bright Quasar Survey, the Bright Quasar Survey, and the Color-Selected sample of the SDSS Fall Equatorial Stripe," Astrophys. J., vol. 699, pp. 800-816, 2009.

[20] A. Schulze, and L. Wisotzki, "Low redshift AGN in the Hamburg/ESO Survey II. The active black hole mass function and the distribution function of Eddington ratios," Astron. Astrophy., vol. 516, pp. A87, 2010.

[21] B. J. Boyle, T. Shanks, and B. A. Petersion, "The evolution of optically selected QSOs-II," Mon. Not. R. Astron. Soc., vol. 235, pp. 935-948, 1988.

[22] S. M. Croom, et al., "The 2dF QSO Redshift Survey XII. The spectroscopic catalogue and luminosity function," Mon. Not. R. Astron. Soc., vol. 349, pp. 1397-1418, 2004.

[23] G. T. Richards, et al., "The 2dF-SDSS LRG and QSO (2SLAQ) Survey: the $\mathrm{z}<2.1$ quasar luminosity function from 5645 quasars to g=21.85," Mon. Not. R. Astron. Soc., vol. 360, pp. 839-852, 2005.

[24] E. Glikman, et al., "The faint end of the quasar luminosity function at $\mathrm{z} \sim 4$ : Implications for ionization of the intergalactic medium and cosmic downsizing," Astrophy. J. Lett., vol. 728, pp. L26, 2011. 
[25] P. Schechter, "An analytic expression for the luminosity function for galaxies, Astrophy. J., vol. 203, pp. 297-306, 1976.

[26] P. Goldschmidt, and L. Miller, "The UVX quasar optical luminosity function and its evolution, Mon. Not. R. Astron. Soc., vol. 293, pp. 107-112, 1998.

[27] S. J. Warren, P. C. Hewett, and P. S. Osmer, "A Wide-Field Multicolor Survey for high-redshift quasars $\mathrm{z} \geq 2.2$. III. The luminosity function," Astrophy. J., vol. 421. pp. 412-433, 2010 .

[28] S. A. Singh, and K. Y. Singh, "The QSOs Luminosity Function from the $2 \mathrm{dF}$ QSO Redshift Survey and the Associated 6dF QSO Redshift Survey at $0.3 \leq \mathrm{z} \leq 2.4$," Advances in Astrophysics, vol. 1, pp. 106-112, 2016.

[29] S. A. Singh, I. A. Meitei, and K. Y. Singh, "Schechter Function Model for the QSO Luminosity Function from the SDSS DR7," International Journal of Astronomy and Astrophysics, vol. 6, pp. 247-253, 2016.

[30] A. Bongiorno, et al., "The VVDS type-1 AGN sample: the faint end of the luminosity function," Astron. Astrophy., vol. 472, pp. 443-454, 2007.

[31] D. G. York, et al., "The Sloan Digital Sky Survey: Technical summary," Astron. J., vol. 120, pp. 1579-1587, 2000.

[32] J. E. Gunn, et al., The $2.5 \mathrm{~m}$ telescope of the Sloan digital Sky Survey,” Astron. J., vol. 182, pp. 2332-2359, 2006.

[33] J. E. Gunn, et al., "The Sloan Digital Sky Survey photometric camera,” Astron. J., vol. 116, pp. 3040-3081, 1998.

[34] M. Fukugita, et al., "The Sloan Digital Sky Survey photometric system,” Astron. J., vol. 111, pp. 1748, 1996.

[35] D. W. Hogg, et al., "A photometricity and extinction monitor at the Apache Point Observatory," Astron. J., vol. 122, pp. 2129-2138, 2001.
[36] Ž. Ivezić, et al., "SDSS data management and photometric quality assessment," Astron. Nachr., vol. 325, pp. 583-589, 2004.

[37] J. R. Pier, et al., "Astrometric calibration of the Sloan Digital Sky Survey,” Astron. J., vol. 125, pp. 1559-1579, 2003.

[38] J. A. Smith, et al., "The u'g'r'ri'z' 350 standard star system," Astron. J., vol. 123, pp. 2121-2144, 2002.

[39] J. B. Oke, and J. E. Gunn, et al., "Secondary standard stars for absolute spectrophotometry," Astrophy. J., vol. 266, pp. 713$717,1983$.

[40] R. H. Lupton, J. E. Gunn, and A. S. Szalay, "A modified magnitude system that produces well-behaved magnitudes, colors, and errors even for signal-to noise ratio measurements," Astron. J., vol. 118, pp. 1406-1410, 1999.

[41] D. P. Schneider, G. T. Richards, P. B. Hall, et al., "The Sloan Digital Sky Survey quasar catalog. V. Seven data release," Astron. J., vol. 139, pp. 2360-2373, 2010.

[42] S. A. Singh, I. A. Singh, and K. Y. Singh, "Optical Luminosity Function of the QSOs Observed with the Sloan Digital Sky Survey Data Release Seven (SDSS DR7)," International Journal of Astronomy and Astrophysics, vol. 4, pp. 474-478, 2014.

[43] G. T. Richards, X. Fan, H. J. Newberg, et al., "Spectroscopic Target Selection in the Sloan Digital Sky Survey: The quasar sample," Astron. J., vol. 123, pp. 2945-2975, 2002.

[44] Y. Shen, and B. C. Kelly, et al., "The demographics of broadline quasars in the mass-luminosity plane. I. Testing FWHMBASED virial black hole masses," Astrophy. J., vol. 746, pp. 169, 2012.

[45] W. H. Press, S. A. Teukolsky, W. T. Vetterling, and B. P. Flannery, "Numerical Recipes in Fortran 77," Cambridge: Cambridge University Press, 1992. 